\title{
Velocities and Linewidths in the Network and Cell Interiors of a Polar Coronal Hole, Compared with Quiet Sun
}

\author{
K.P. Raju ${ }^{1}$ - B.J.I. Bromage ${ }^{2}$ \\ (c) Springer $\bullet \bullet \bullet \bullet$
}

\begin{abstract}
The relative Doppler velocities and linewidths in a polar coronal hole and the nearby quiet-Sun region have been obtained from the Solar and Heliospheric Observatory (SOHO)/Coronal Diagnostic Spectrometer (CDS) observations using emission lines originating at different heights in the solar atmosphere from the lower transition region (TR) to the low solar corona. The observed region is separated into the network and the cell interior and the behavior of the above parameters were examined in the different regions. It has been found that the histograms of Doppler velocity and width are generally broader in the cell interior as compared to the network. The histograms of Doppler velocities of the network and cell interior do not show significant difference in most cases. However, in the case of the quiet Sun, the Doppler velocities of the cell interior are more blueshifted than those of the network for the lowermost line He II 304 $\AA$, and an opposite behavior is seen for the uppermost line Mg IX $368 \AA$. The histograms of line width show that the network-cell difference is more prominent in the coronal hole. The network has significantly larger linewidth than the cell interior for the lowermost TR line He II $304 \AA$ for the quiet Sun. For coronal hole, this is true for the three lower TR lines He II $304 \AA$, O III $599 \AA$, and O v $630 \AA$. Also obtained are the correlations between the relative Doppler velocity and the width. A mild positive correlation is found for the lowermost transition region line He II $304 \AA$ which further decreases or become insignificant for the intermediate lines. For the low coronal line, Mg IX $368 \AA$, the correlation becomes strongly negative. This could be due to the presence of standing or propagating waves from the lower to the upper solar atmosphere. The results may have implications for the generation of the fast solar wind and coronal heating.
\end{abstract}

Keywords: Coronal Holes; Transition Region; Spectrum, Ultraviolet

\footnotetext{
1 Indian Institute of Astrophysics, Bangalore-560034, India email: kpr@iiap.res.in

2 Jeremiah Horrocks Institute,

University of Central Lancashire, Preston, PR1 2HE, UK

email: bjibromage@uclan.ac.uk
} 


\section{Introduction}

The chromospheric network, the bright emission network seen in the chromospheric lines such as $\mathrm{Ca}$ II and $\mathrm{H} \alpha$ lines, represents the boundaries of the supergranulation cells (Simon and Leighton, 1964). The convective motions in the cells sweep small flux tubes to the edges of the cells, resulting in magnetic concentrations and enhanced emission there. The EUV emission network is essentially the continuation of the chromospheric network in the transition region (Brueckner and Bartoe, 1974; Reeves et al., 1974). The network slowly disintegrates and becomes indistinguishable from the surroundings in the corona.

The morphological properties and the evolution of the network are different in the quiet Sun and the coronal hole. Skylab observations in the 1970s have shown that the network emission is weakened in coronal holes as compared to quiet-Sun region (Huber et al., 1974). Gallagher et al. (1998) have obtained the intensity contrast of the network with respect to the internetwork for different lines in the quiet Sun, and the maximum was found for the O v $629.73 \AA$ line whose formation temperature is about $0.25 \mathrm{MK}$. Raju (2010) obtained the intensity contrast of the network for different lines in both quiet Sun and coronal hole. The contrast, in general, is lower for the coronal hole as compared to the quiet Sun, but becomes equal in the upper transition region. The maximum contrast for both the regions was found at about 0.2 MK. These results seem to suggest that the Doppler velocities and widths could also be different in the network and the cell interior and also there are possible variations between quiet Sun and coronal hole.

It has been known that there is a systematic difference between coronal hole and the quiet Sun in terms of intensity, velocity, and width (Raju, 2009). Judge, Carlsson, and Wilhelm (1997) report that Transition region (TR) lines show more redshift in the network than in the inter-network and also there is a correlation between line intensity brightenings and increased redshift. Gontikakis et al. (2001) also find that velocity distribution is different for network and inter-network with network having more redshift with lower standard deviation. However, Hassler et al. (1999) find that plasma outflow in coronal hole originates predominantly along the network boundaries. Popescu, Doyle, and Xia (2004) report a correlation between network intensity and Doppler velocity for the O III line.

In the present article, spectroscopic data from Solar and Heliospheric Observatory (SOHO)/Coronal Diagnostic Spectrometer (CDS) were used to examine the variations of Doppler velocities and emission linewidth in the network and the cell interior in a polar coronal hole $(\mathrm{PCH})$ and the quiet Sun region outside. Observations were made in five different emission lines whose formation temperatures vary from $0.08-0.95 \mathrm{MK}$ and hence represent the lower transition region to the inner corona. In particular, two aspects have been examined; i) evolution of the network in the solar atmosphere, and ii) the correlation between Doppler velocity and linewidth in the different regions. 


\section{Data and Analysis}

SOHO/CDS observations made during the Whole Sun Month (August - September 1996) have been used. Details of the observations and data reduction have been given by Bromage et al. (2000) and Raju (2009). Only five strong lines were selected for the present analysis. Details of the emission lines were obtained from CHIANTI (Dere et al., 1997; Landi et al., 2006) and may be seen in Table 1. The CDS field of view is $240^{\prime \prime} \times 60^{\prime \prime}$, which is a combination of three rasters. The spatial resolution is approximately $4^{\prime \prime}$ but a running averaging of five nearby pixels has reduced this to about $20^{\prime \prime}$ and the overall temporal resolution to about an hour. 14 datasets from the north polar region near the central meridian are used in this study. The Normal Incidence Spectrometer (NIS) data suffer from instrumental trends due to the rotation and tilt effects (Brooks and Bewsher, 2006). These are corrected as described by Rajul (2009).

The spectra obtained from the CDS windows were fitted with single or multiple Gaussians depending upon the number of lines present in the window using the routine CFIT-BLOCK in Solar Software (SSW). The individual line profiles were always fitted with a single Gaussian. The He window has two strong lines, He II at $304 \AA$ (second order at $607.56 \AA$ ) and O IV at $608.31 \AA$, and hence a double Gaussian fit was used. The O III $599 \AA$ and O v $630 \AA$ windows were fitted with single Gaussian. The Ne VI window was fitted with a double Gaussian to fit Ne VII $561.7 \AA$ and Ne VI $562.8 \AA$. The Mg IX window was fitted with a double Gaussian to fit Mg VII $367.7 \AA$ and Mg IX $368 \AA$. It may be noted that the zero point in velocity is kept equal to the most probable value in the data, and hence the Doppler velocities in the present analysis are relative Doppler velocities with respect to the coronal rest frame. Only those line profiles with a signal-to-noise ratio greater than ten were selected for the analysis. With this, the estimated errors are $2 \mathrm{~km} \mathrm{~s}^{-1}$ in velocity and $0.03 \AA$ in width.

Table 1. Details of the emissionline parameters. Note that He line is observed in the second order.

\begin{tabular}{clcl}
\hline No. & Ion & $\begin{array}{c}\lambda \\
{[\AA]}\end{array}$ & $\begin{array}{l}\mathrm{T} \\
{[\mathrm{MK}]}\end{array}$ \\
\hline 1 & He II & 303.78 & 0.083 \\
2 & O III & 599.59 & 0.11 \\
3 & O V & 629.73 & 0.25 \\
4 & Ne VI & 562.80 & 0.43 \\
5 & Mg IX & 368.06 & 0.95 \\
\hline
\end{tabular}

The coronal hole and the quiet Sun were identified in the images on the basis of the intensity of the $\mathrm{Mg}$ x $625 \AA$ line. The network and the cell interior are defined on the basis of the intensity distribution of the O v $629.73 \AA$ line. Those points for which the intensity is above two-thirds of the distribution are taken as network. Reeves (1976) took the average intensity to distinguish the network 
and the cell interior, whereas some authors (Xia, Marsch, and Wilhelm, 2004) have taken the two-thirds criterion. We find that the results remain the same irrespective of the two criteria.

\section{Results}

Histograms of relative Doppler velocities in the network and the cell interior in the quiet Sun and coronal hole in different emission lines are given in Figure 1. The bin width is $2 \mathrm{~km} \mathrm{~s}^{-1}$. Also obtained are the mean, its error, and the standard deviation of each distribution, which are given in Table 2. An examination of the figure and the table reveals that the histograms of Doppler velocities of the cell interior are broader than that of the network except in the case of $\mathrm{He}$ line in the coronal hole. The histograms of Doppler velocities of the network and cell interior do not show significant difference in most cases. However, in the case of the quiet Sun, the Doppler velocities of the cell interior are more blueshifted than that of the network for the lowermost line He II $304 \AA$, and an opposite behavior is seen for the uppermost line Mg IX $368 \AA$ where it is the velocities of the network that are more blueshifted than that of the cell interior. For the intermediate lines from the quiet Sun, as well as for the lines from the coronal hole, the behavior is inconclusive because of the large uncertainties.

Histograms of linewidths are given in Figure 2. The bin width is $0.01 \AA$. The mean, error, and standard deviation are given in Table 3 . It can be seen that the histograms of the cell interior are broader than those of the network in most of the cases. The exceptions are the $\mathrm{O} \mathrm{V}$ and $\mathrm{Mg}$ IX lines in the quiet Sun and the He II line in the coronal hole. Also the network-cell interior difference is more prominent in the coronal hole. The network has significantly larger linewidth than the cell interior for the lowermost TR line He II $304 \AA$ for the quiet Sun. For a coronal hole, this is true for the three lower TR lines He II $304 \AA$, O III $599 \AA$, and O v $630 \AA$. This shows that the network-cell interior difference disappears faster in the quiet Sun.

The correlations between Doppler velocity and linewidth for the individual points in the different regions are shown in Figure 3. A straight-line fit is given to show the overall behavior. Also given are the correlation coefficient and the probability that the correlation can arise from two random distributions.

The correlation coefficients obtained from Figure 3 are plotted against the formation temperatures of different lines in Figure 4. A mild positive correlation is found for the lowest TR line He II $304 \AA$. The correlation reduces or become insignificant for the lower transition region lines O III $599 \AA$ and O V $630 \AA$. The correlation is completely insignificant for the upper TR line Ne VI $562.8 \AA$. For the low coronal line Mg IX $368 \AA$, the correlation becomes strongly negative. It may also be seen that the correlation coefficients do not show any significant difference between the different regions.

\section{Discussion}

Observations in recent years have shown that a coronal hole has larger blueshifts and linewidths as compared to a quiet-Sun region (Banerjee et al., 1998) Raju et 

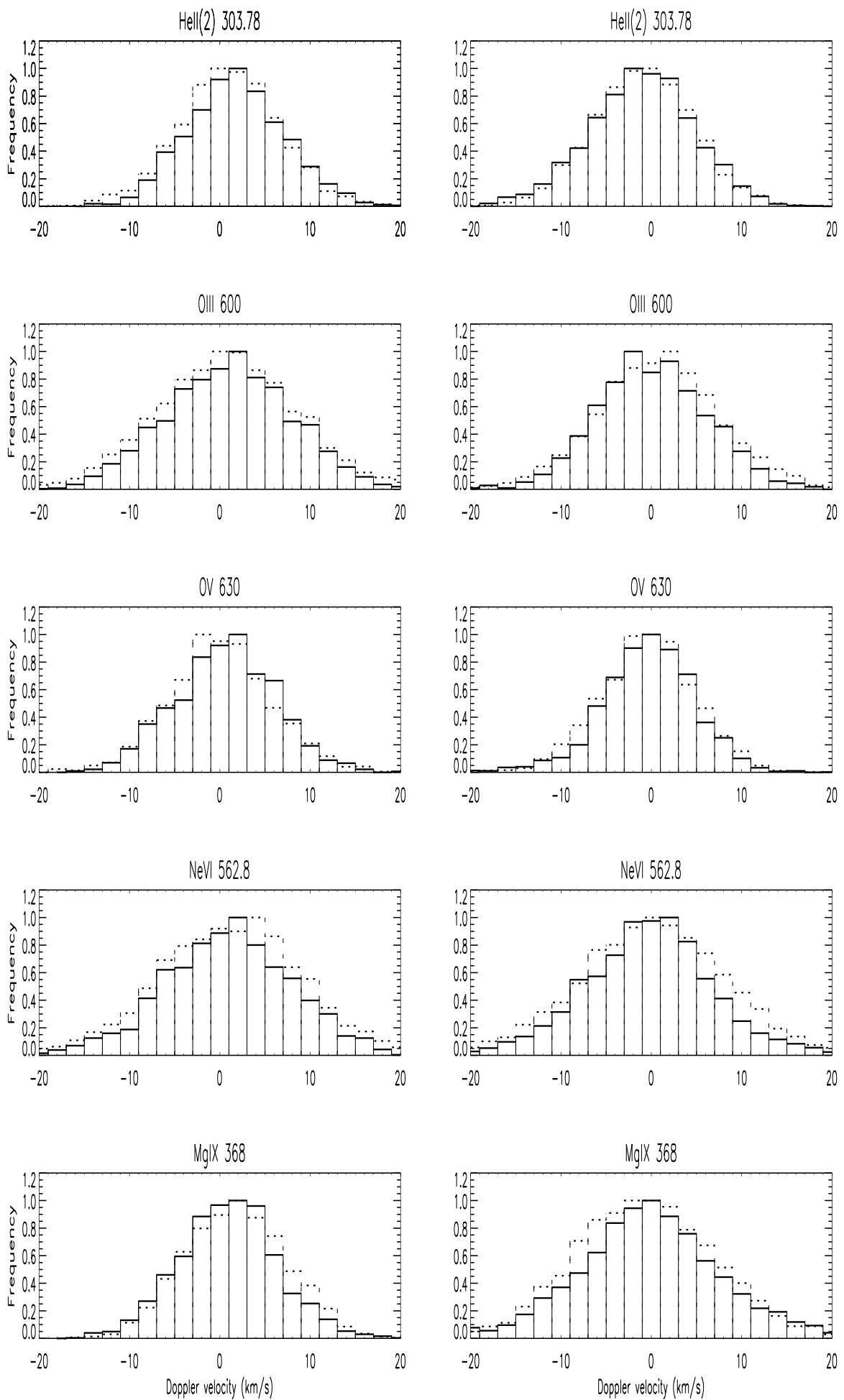

Figure 1. Histograms of Doppler velocities for different emission lines. The left panels represent quiet Sun and right panels represent coronal hole. Solid line represents the network and the dotted line represents cell interior. 

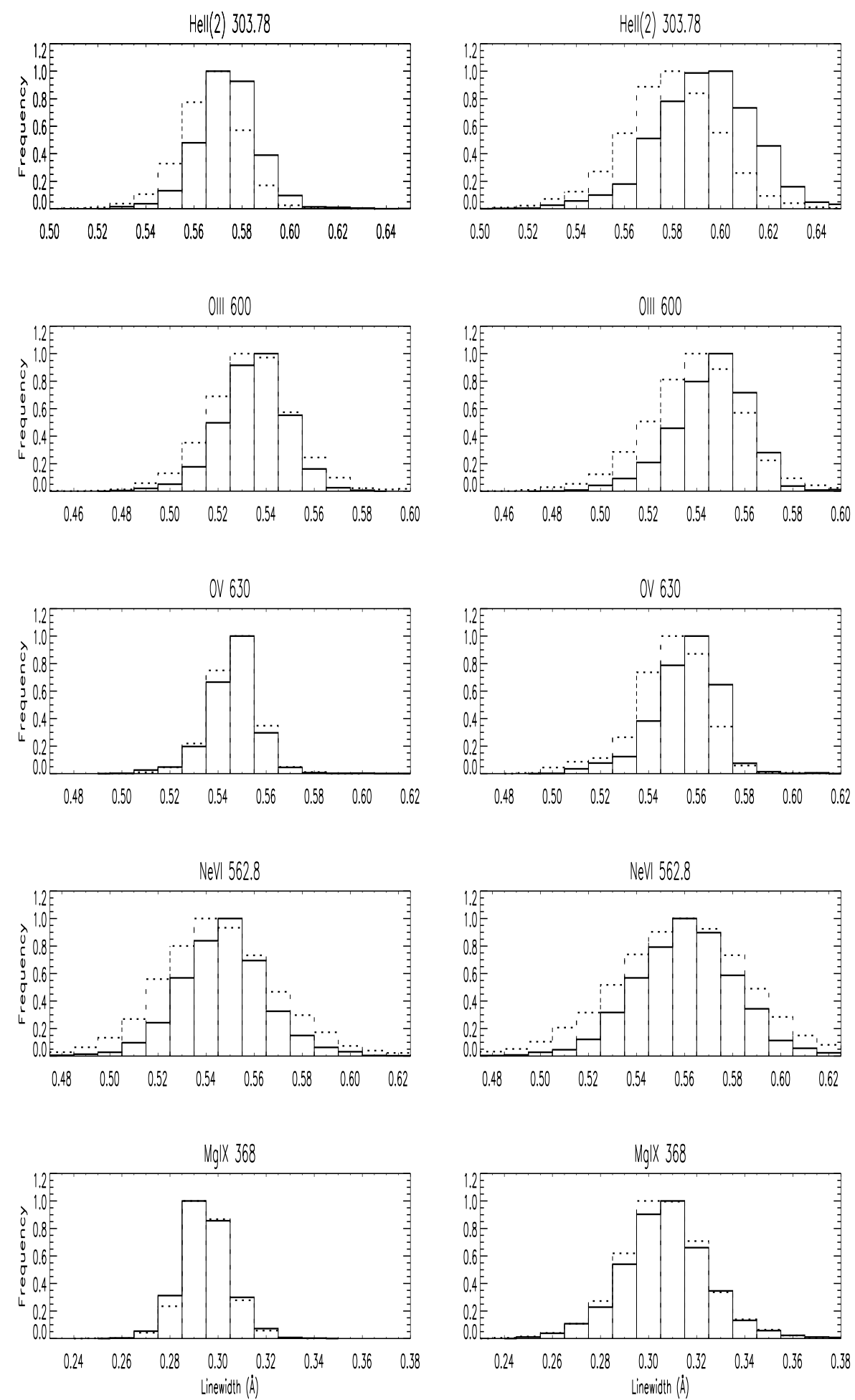

Figure 2. Histograms of linewidths for different emission lines. The left panels represent quiet Sun and right panels represent coronal hole. Solid line represents the network and the dotted line represents cell interior. 

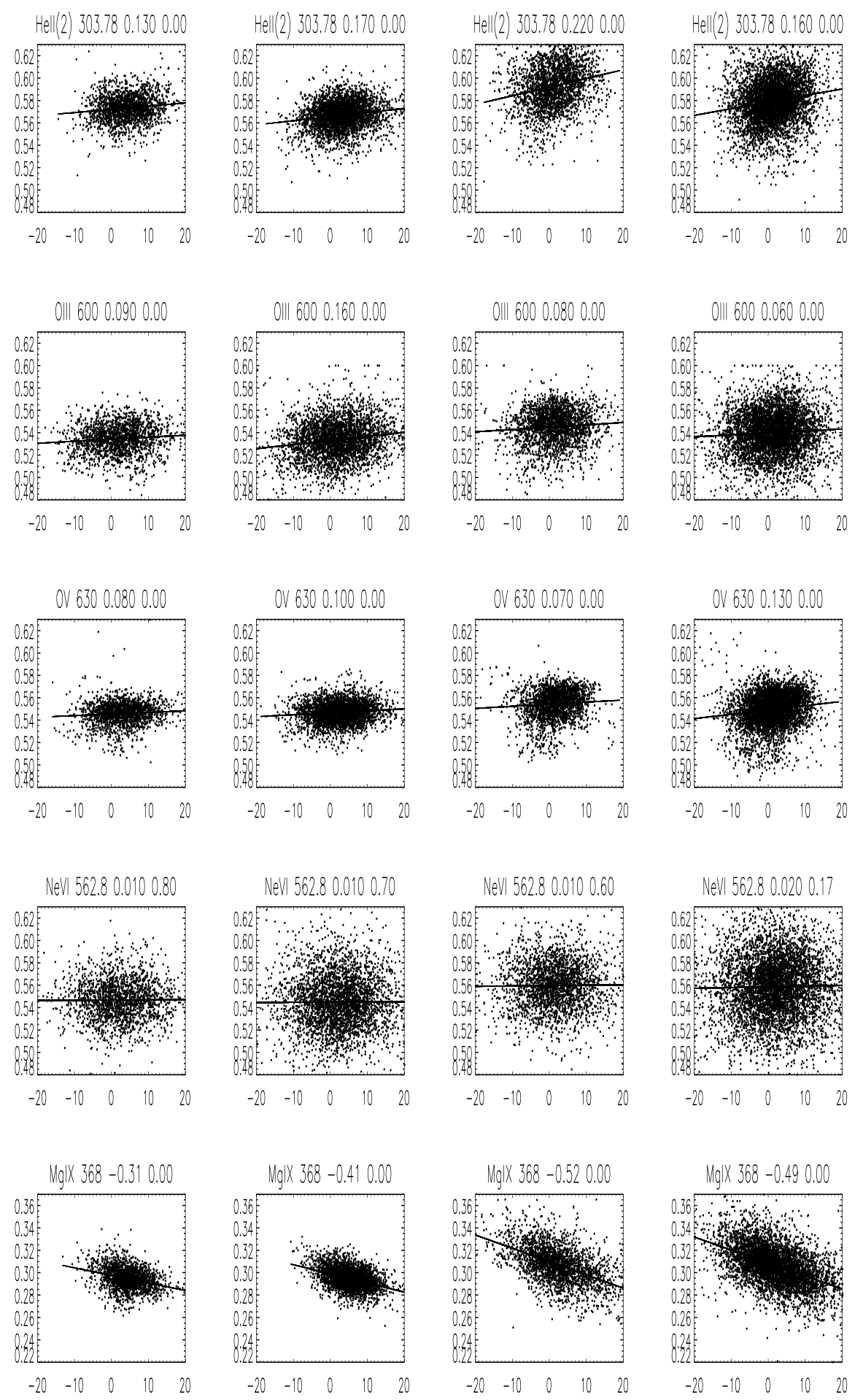

Figure 3. Linewidths $[\AA]$ are plotted against Doppler velocities $\left[\mathrm{km} \mathrm{s}^{-1}\right]$ for different emission lines. The line details, correlation coefficient and the probability that the correlation can arise from two random distributions are given above each panel. From left to right; First panel represents network in quiet Sun, second represents cell interior in quiet Sun, third represents network in coronal hole, and fourth represents cell interior in coronal hole. 
Table 2. Details of velocity histograms (given in Figure 1). Mean of velocity distribution [vel] in $\mathrm{km} \mathrm{s}^{-1}$, its error, and standard deviation [stdv] of the network and cell interior in the quiet Sun and coronal hole are given in different columns.

\begin{tabular}{|c|c|c|c|c|c|c|c|c|}
\hline \multirow[t]{3}{*}{ No. } & \multicolumn{4}{|c|}{ QS } & \multicolumn{4}{|c|}{$\mathrm{CH}$} \\
\hline & \multicolumn{2}{|c|}{ network } & \multicolumn{2}{|l|}{ cell } & \multicolumn{2}{|c|}{ network } & \multicolumn{2}{|l|}{ cell } \\
\hline & vel & stdv & vel & stdv & vel & stdv & vel & stdv \\
\hline 1 & $1.65 \pm 0.30$ & 4.95 & $0.99 \pm 0.23$ & 5.12 & $-1.02 \pm 0.69$ & 5.29 & $-0.90 \pm 0.41$ & 5.14 \\
\hline 2 & $0.94 \pm 0.37$ & 6.07 & $0.59 \pm 0.29$ & 6.38 & $0.08 \pm 0.73$ & 5.57 & $0.59 \pm 0.47$ & 5.86 \\
\hline 3 & $0.54 \pm 0.31$ & 5.17 & $0.04 \pm 0.24$ & 5.25 & $-0.27 \pm 0.62$ & 4.72 & $-0.43 \pm 0.40$ & 4.96 \\
\hline 4 & $0.80 \pm 0.37$ & 6.08 & $0.88 \pm 0.29$ & 6.48 & $-0.32 \pm 0.78$ & 5.98 & $0.08 \pm 0.53$ & 6.56 \\
\hline 5 & $0.83 \pm 0.31$ & 5.05 & $1.59 \pm 0.24$ & 5.19 & $-0.17 \pm 0.83$ & 6.32 & $-0.59 \pm 0.52$ & 6.43 \\
\hline
\end{tabular}

Table 3. Details of linewidth histograms (given in Figure 2). Mean of linewidth distribution [wid] in $\AA$, its error, and standard deviation [stdv] of the network and cell interior in the quiet Sun and corona hole are g.ven in different columns.

\begin{tabular}{|c|c|c|c|c|c|c|c|c|}
\hline \multirow[t]{3}{*}{ No. } & \multicolumn{4}{|c|}{ QS } & \multicolumn{4}{|c|}{$\mathrm{CH}$} \\
\hline & \multicolumn{2}{|c|}{ network } & \multicolumn{2}{|l|}{ cell } & \multicolumn{2}{|c|}{ network } & \multicolumn{2}{|l|}{ cell } \\
\hline & wid & stdv & wid & stdv & wid & stdv & wid & stdv \\
\hline 1 & $0.573 \pm 0.001$ & 0.012 & $0.566 \pm 0.001$ & 0.013 & $0.593 \pm 0.003$ & 0.020 & $0.579 \pm 0.002$ & 0.019 \\
\hline 2 & $0.534 \pm 0.001$ & 0.013 & $0.533 \pm 0.001$ & 0.017 & $0.545 \pm 0.002$ & 0.015 & $0.539 \pm 0.002$ & 0.019 \\
\hline 3 & $0.546 \pm 0.001$ & 0.010 & $0.546 \pm 0.001$ & 0.009 & $0.555 \pm 0.001$ & 0.013 & $0.548 \pm 0.001$ & 0.015 \\
\hline 4 & $0.547 \pm 0.001$ & 0.018 & $0.545 \pm 0.001$ & 0.024 & $0.560 \pm 0.003$ & 0.020 & $0.558 \pm 0.002$ & 0.028 \\
\hline 5 & $0.294 \pm 0.001$ & 0.010 & $0.295 \pm 0.001$ & 0.009 & $0.307 \pm 0.002$ & 0.017 & $0.306 \pm 0.001$ & 0.018 \\
\hline
\end{tabular}

al., 2000; Stucki et al., 2000). This has been understood as due to the origin of fast solar wind in the coronal hole (Harra, 2012). The network and cell interior show large difference in their intensities (Reeves et al., 1974; Gallagher et al., 1998; Raju, 2010) and widths (Figure 2) and therefore we expect large differences in their velocities, which is not seen. However, the pattern of difference seen in the lower-TR line He II $304 \AA$ and the low-coronal line Mg IX $368 \AA$ in the quiet Sun is interesting and is being reported for the first time. This also poses challenges for interpretation. Judge, Carlsson, and Wilhelm (1997) and Gontikakis et al. (2001) report that the lower-TR lines show more redshift in the network than in the internetwork in the quiet Sun, which agrees with our result. Hassler et al. (1999) observe large blueshifts in the network in coronal hole in the Ne VIII 770 $\AA$ line which is formed at the base of the solar corona. Our results on the low coronal line $\mathrm{Mg}$ Ix $368 \AA$ from the coronal hole are inconclusive. Before going further, these results need to be verified.

The larger linewidths in the network as compared to the cell interior are expected because the network is hotter than the latter. The results also show that the evolution of the network is faster in the quiet Sun than in the coronal hole. This is expected to be due to the difference in the thickness of the TR which is five times large in the coronal hole (Huber et al., 1974). 


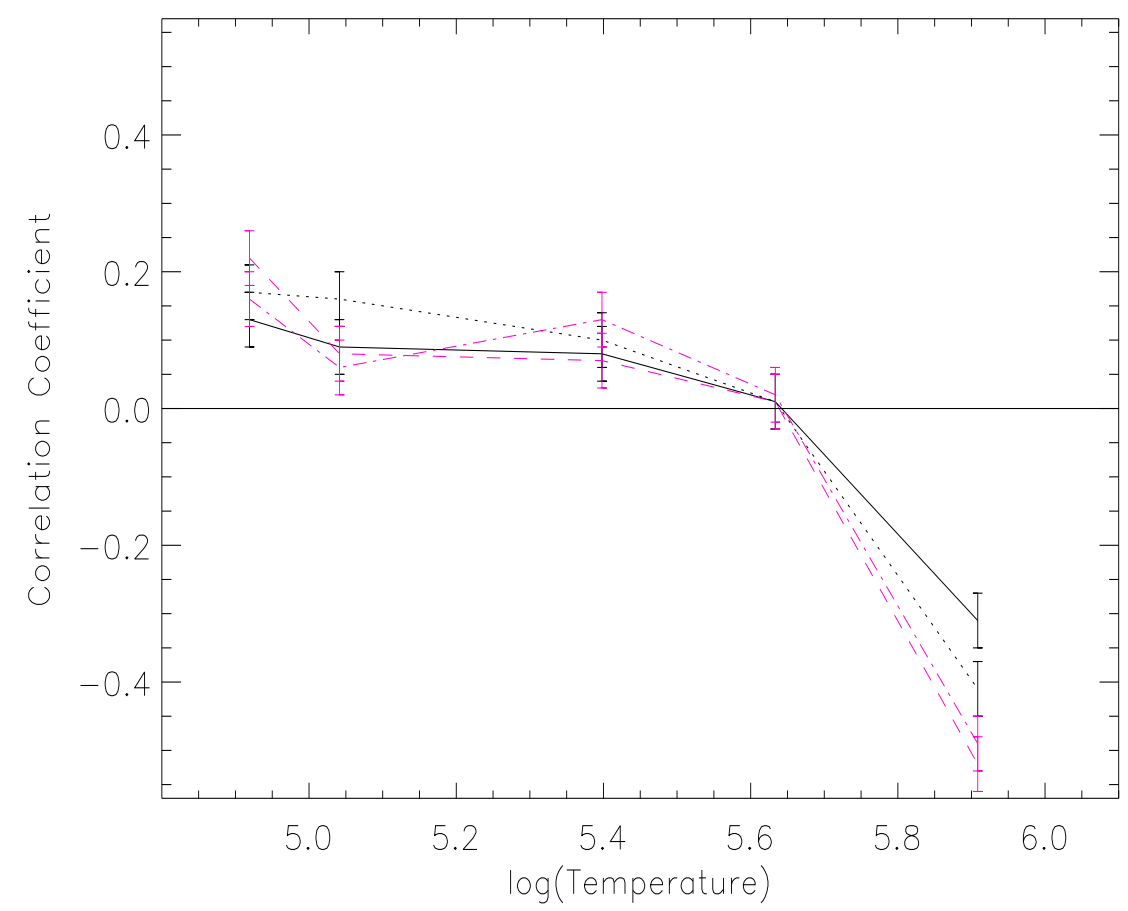

Figure 4. Correlation coefficients plotted against log of formation temperatures of the different lines. The solid line represents network in quiet Sun, dots represent cell interior in quiet Sun, dashes represent network in coronal hole, and dash-dots represent cell interior in coronal hole.

Plumes are known to have smaller linewidths and velocities compared to interplumes (Wilhelm et al., 1998). The excess width in the network of the coronal hole raises some questions regarding the network-origin of plumes. If plumes are indeed the extensions of the network, then we might expect lower widths in the network. The results on the velocity distributions are, however, inconclusive.

The change of sign of the correlation coefficients of linewidth and velocity for lines from transition region to the corona is being reported for the first time, although some evidence of this can be seen by Xia, Marsch, and Wilhelm (2004). In their work with Solar Ultraviolet Measurements of Emitted Radiation (SUMER) data, the Si II $1533 \AA$ line shows a positive correlation while the O VI $1038 \AA$ line shows a negative correlation and the behavior of other lines is ambiguous. The behavior of correlation coefficients could be due to the presence of standing or propagating waves from the lower to the upper solar atmosphere. For example, Alfvèn waves can cause simultaneous variations in width and velocity. Alfvèn waves are known to be one of the candidates for coronal heating. 


\section{Conclusions}

We have obtained the Doppler velocities and emission linewidths in a coronal hole and the nearby quiet Sun in five different emission lines originating at different heights in the solar atmosphere from the lower TR to the inner corona. The behavior of the velocities and widths in the network and the cell interior in the coronal hole and quiet Sun were examined. Histograms of Doppler velocity and width are generally broader in the cell interior as compared to the network. The histograms of Doppler velocities of the network and cell interior do not show significant differences in most cases with exceptions in the lower-TR line He II $304 \AA$ and the low-coronal line Mg IX $368 \AA$ in the quiet Sun. Doppler velocities of the cell interior are more blueshifted than that of the network for the He line and an opposite behavior is seen for the $\mathrm{Mg}$ line. The histograms of line width show that the network-cell difference is more prominent in the coronal hole and the network-cell merger happens faster in the quiet Sun. A mild positive correlation between the relative Doppler velocity and the linewidth is found for the lowermost transition region line He II $304 \AA$, which further reduces or become insignificant for the intermediate lines, and becomes strongly negative for the low coronal line, Mg IX $368 \AA$. This could be due to the presence of standing or propagating waves from the lower to the upper solar atmosphere

Acknowledgements Data are provided courtesy of SOHO/CDS consortium. SOHO is a project of international cooperation between ESA and NASA. 


\section{References}

Banerjee, D., Teriaca, L., Doyle, J.G., Wilhelm, K.:1998, Broadening of SI VIII lines observed in the solar polar coronal holes. Astron. Astrophys. 339, 208. ADS 1998A\&A...339..208B

Bromage, B.J.J., Alexander, D., Breen, A., Clegg, J.R., Del Zanna, G., DeForest, C., Dobrzycka, D., Gopalswamy, N., Thompson, B., Browning, P.K.: 2000, Structure of a Large low-Latitude Coronal Hole. Solar Phys. 193, 181. ADS 2000SoPh..193..181B doi $10.1023 /$ A.1005209725885

Brooks, D.H., Bewsher, D.: 2006, On Deriving Plasma Velocity Information from CDS/NIS Observations: Application to the Dynamics of Blinkers. Solar Phys. 234, 257. ADS 2006SoPh..234..257B, doi 10.1007/s11207-006-0096-3

Brueckner, G., Bartoe, J.-D.: 1974, The fine structure of the solar atmosphere in the far ultraviolet. Solar Phys. 38, 133. ADS 1974SoPh...38..133B doi 10.1007/BF00161831

Dere, K.P., Landi, E., Mason, H.E., Monsignori Fossi, B.C., Young, P.R.: 1997, CHIANTI - an atomic database for emission lines. Astrophys. J. Suppl. Ser. 125, 149. ADS 1997A\&AS..125..149D doi 10.1051 aas:1997368

Gallagher, P.T., Phillips, K.J.H., Harra-Murnion, L.K., Keenan, F.P.: 1998, Properties of the quiet Sun EUV network. Astron. Astrophys. 335, 733. ADS 1998A\&A...335..733G

Gontikakis, C., Dara, H.C., Alissandrakis, C.E., Zachariadis, T.G., Vial, J.-C.: 2001, Dopplershifts in the solar transition region. Astron. Astrophys. 378, 257. ADS 2001A\&A...378..257G doi 10.1051/0004-6361:20011231

Harra, L.: 2012, In: Bellot Rubio, L.R., Reale, F., Carlsson, M. (eds), 4th Hinode Meeting: Unsolved Problems and New Insights, CS-455, Astron. Soc. Pac. 315.

Hassler, D.M., Dammasch, I.E., Lemaire, P., Brekke, P., Curdt, W., Mason, H.E., Vial, J.-C., Wilhelm, K.: 1999, Solar Wind Outflow and the Chromospheric Magnetic Network. Science 283, 810. ADS 1999Sci...283.810H, doi 10.1126/science.283.5403.810

Huber, M.C.E., Foukal, P.V., Noyes, R.W., Reeves, E.M., Schmahl, E.J., Timothy, J.G., Vernazza, J.E., Withbroe, G.L.: 1974, Extreme-ultraviolet observations of coronal holes - Initial results from SKYLAB. Astrophys. J. Lett. 194, L115. ADS 1974ApJ...194L.115H. doi $10.1086 / 181682$

Judge, P., Carlsson, M., Wilhelm, K.: 1997, SUMER Observations of the Quiet Solar Atmosphere: The Network Chromosphere and Lower Transition Region. Astrophys. J. Lett. 490, L195. ADS 1997ApJ...490L.195J, doi 10.1086/311037

Landi, E., Del Zanna, G., Young, P.R., Dere, K.P., Mason, H.E., Landini, M.: 2006, CHIANTI-An Atomic Database for Emission Lines. VII. New Data for X-Rays and Other Improvements. Astrophys. J. Suppl. Ser. 162, 261. ADS 2006ApJS..162..261L doi $10.1086 / 498148$

Popescu, M.D., Doyle, J.G., Xia, L.D.: 2004, Network boundary origins of fast solar wind seen in the low transition region? Astron. Astrophys. 421, 339. ADS 2004A\&A...421..339P doi 10.1051/0004-6361:20034348

Raju, K.P., Sakurai, T., Ichimoto, K., Singh, Jagdev: 2000, The Physical Conditions in a Polar Coronal Hole and Nearby Regions from Norikura and SOHO Observations. Astrophys. J. 543, 1044. ADS 2000ApJ...543.1044R doi $10.1086 / 317143$

Raju, K.P.: 2009, Relative Velocities and Linewidths in a Coronal Hole and Outside. Solar Phys. 255, 119. ADS 2009SoPh..255..119R doi 10.1007/s11207-008-9309-2

Raju, K.P.: 2010, Intensity Distribution and Contrast of the Solar EUV Network. Solar Phys. 262, 61. ADS 2010SoPh..262...61R doi 10.1007/s11207-010-9514-7

Reeves, E.M., Foukal, P.V., Huber, M.C.E., Noyes, R.W., Schmahl, E.J., Timothy, J.G., Vernazza, J.E., Withbroe, G.L.: 1974, Observations of the Chromospheric Network: Initial Results from the Apollo Telescope Mount. Astrophys. J. Lett. 188, 27. ADS 1974 ApJ...188L..27R, doi $10.1086 / 181423$

Reeves, E.M.: 1976, The EUV chromospheric network in the quiet sun. Solar Phys. 46, 53. ADS 1976 SoPh...46...53R, doi $10.1007 /$ BF00157554

Simon, G.W., Leighton, R.B.: 1964, Velocity Fields in the Solar Atmosphere. III. Large-Scale Motions, the Chromospheric Network, and Magnetic Fields. Astrophys. J. 140, 1120. ADS 1964ApJ...140.1120S doi $10.1086 / 148010$

Stucki, K., Solanki, S. K., Schühle, U., Rüedi, I., Wilhelm, K., Stenflo, J. O., Brković, A., Huber, M. C. E.: 2000, Comparison of far-ultraviolet emission lines formed in coronal holes and the quiet Sun. Astron. Astrophys. 363, 1145. ADS 2000A\&A...363.1145S 
Wilhelm, K., Marsch, E., Dwivedi, B.N., Hassler, D.M., Lemaire, P., Gabriel, A.H., Huber, M.C.E.: 1998, The Solar Corona above Polar Coronal Holes as Seen by SUMER on SOHO. Astrophys. J. 500, 1023. ADS 1998 ApJ...500.1023W doi $10.1086 / 305756$

Xia, L.D., Marsch, E., Wilhelm, K.: 2004, On the network structures in solar equatorial coronal holes. Observations of SUMER and MDI on SOHO. Astron. Astrophys. 424, 1025. ADS 2004A\&A...424.1025X doi 10.1051/0004-6361:20047027. 